\title{
Impact of aqueous bark extract of Terminalia arjuna on hematological and immunological profile variables of albino rats
}

\author{
Priyanka Sagar ${ }^{1 *}$, Manoranjan Prasad Sinha ${ }^{1}$, Manoj Kumar ${ }^{2}$ \\ ${ }^{1}$ Department of Zoology, Ranchi University, Ranchi - 834 008, Jharkhand, INDIA \\ ${ }^{2}$ Department of Zoology, St. Xavier's College, Ranchi - 834001 \\ *e-mail: priyankasagar8@gmail.com
}

\begin{abstract}
The effect of aqueous extract of bark of Terminalia arjuna on some hematlogical and immunological parameters in rats during a seven-day administration of the doses of $250 \mathrm{mg} / \mathrm{kg}$ and $500 \mathrm{mg} / \mathrm{kg}$ body weight orally was investigated. The results showed significant increase in total leucocyte count, monocytes, eosinophil, monocytes, hemoglobin, mean corpuscular volume, mean corpuscular hemoglobin, and mean corp. hemo. Conc, and platelet count in both the cases, when it was treated with low doses and high doses but in neutrophil, hematocrit significantly increased in case of low doses and reduced significantly with high doses and lymphocyte reduced with low doses and significantly increased in case of high doses. In Immunology, the IGG count showed no significant change in low dose, high dose as compared to control and non-significant changes when low dose compared to high dose. The IGM count showed significant changes in low dose, as compared to control; increased significantly in high dose, compared to control and non-significant changes observed in low dose as compared to high dose. The IGA count showed no significant changes when low dose, high dose was compared to control but increased significantly, when low dose compared to high dose. The results of this study suggest that the extracts showed beneficial effects on hematology and immunity related disorders and can be used in medicinal formulations.
\end{abstract}

Key words: IgG, IgA, IgM, Terminalia arjuna, Hematological, Immunological

\section{INTRODUCTION}

Natural products are part of our everyday life. Right from the inception, India has a rich heritage of usage of Ayurvedic and Herbal medicines. Herbal products have just recently started rising on the horizon of alternative system of medicine. Ayurved and Herbal products were being practiced and used all over the world for many years but have only recently started getting legal acceptance in many countries in the world as alternative system of medicine. India is called as "Botanical Garden of the world" as it is largest producer of medicinal herbs [1].
In the present study the aqueous bark extract of $T$. arjuna was prepared in a manner that is followed by tribals and rural people for their consumption as medicine.

Terminalia arjuna belonging to the family Combretaceae is an important medicinal plant widely used by the tribal and rural people for alleviating angina and other cardiovascular conditions for over three centuries in India. Experimental studies have revealed that the stem

bark exerts significant inotropic and hypotensive effect, increasing coronary artery flow and protecting myocardium against ischemic damage [2]. 
Hematology is a subspecialty of internal medicin e and is concerned with the nature, function, and diseases of the blood. Hematology uses highly d eveloped technology to make specific diagnoses and treat illnesses occurring in all organ systems. The blood is a vital fluid, which contains the Red Blood Cell (RBC), White blood cells (WBC) and platelets suspended in the serum in homeostatic concentrations [3].

The Blood is important for pulmonary and tissue respiration, as a medium of endocrine and neurohumoral transmissions, biotransformation and metabolic excretion [4], nutritional and immunological processes, as well as homeostatic responses. The laboratory determination of blood products and parameters for the purpose of disease diagnosis is highly accurate, sensitive and reliable; and remained the bed-rock of ethical and rational research, disease diagnosis; prevention and treatment [3]

\section{MATERIALS AND METHODS}

\section{Terminalia arjuna}

Terminalia arjuna is the immensely colossal size deciduous tree. The height of the arjuna tree reaches up to $60-85$ feet. It is the evergreen tree with the yellow flowers and conical leaves. It has a smooth gray bark. Fruit is 2.5-3.5 cm long, fibrous woody, glabrous with 5 hard wings, striated with numerous curved veins. It has a buttressed trunk and an astronomical spreading crown from which the branches drop downwards. Its leaves are dull green above and pale brown beneath $T$._arjuna flowers between March to June and fruits between September to November [5].

\section{Collection of materials:}

Plant sample of $T$. arjuna was collected from Ranchi in May 2014 and a plant sample was submitted to the Department of zoology, Ranchi University, Ranchi for identification. Barks were shade dried for few days. The dried barks were then ground in coarse powder using high capacity grinding machine which was then stored in air-tight container with necessary markings for identification and kept in cool, dark and dry place for the investigation [6-8].

\section{Preparation of Extract:}

$50 \mathrm{~g}$ of the $T$. arjuna powder was weighed accurately and subjected to extraction in a soxhlet apparatus at $37^{\circ}$ temperature using $\sim 250 \mathrm{~mL}$ distilled water. The extract obtained was filtered, concentrated in rotary flash evaporator and maintained at $45^{\circ} \mathrm{C}$ the percentage yield of each extract were calculated and the aqueous extracts were stored in air tight containers at room temperature for further studies [9-10].

\section{Experimental Animals:}

Male Albino rats (175-200 g) were used in the study. They were maintained under standard laboratory conditions at ambient temperature of $25 \pm 2^{\circ} \mathrm{C}$ and $50 \pm 1.5 \%$ relative humidity with a $12-\mathrm{h}$ light/12-h dark cycle. Animals were fed with a commercial pellet diet and water ad libitum. The experiments were performed after prior approval of the study protocol by the institutional animal ethics committee of Ranchi University, Ranchi (process no. 46, page no.137) [11]. 


\section{Collection of blood sample:}

By the end of each experimental period, the rats were reweighed, starved for 24 hours and sacrificed under chloroform anesthesia. $5 \mathrm{~mL}$ of blood was collected from each animal by cardiac puncture using sterile needle and syringe. Part of the blood sample was put into test tubes and allowed to clot for 30 minutes before centrifuging at $800 \mathrm{~g}$ (Wisperfuge, 1384, Samson, Holland) for 5 minutes. Thesupernatant was used for the lipid analysis. The remaining blood sample was put in an EDTA bottles for hematological determinations.

\section{Analytic procedure:}

Estimation of total cholesterol, HDL cholesterol, LDL cholesterol and triglycerides was done by cholesterol oxidase - phenol amino antipyrine method [12].

\section{RESULTS AND DISCUSSION}

The present study evaluated the Hematological and immunological activities of aqueous extract from Terminalia arjuna bark in experimental animal. The result indices are presented in table 1.

Table1: Effects of Terminalia arjuna bark extracts on Hematological profile of male albino wistar rats at $7^{\text {th }}$ days (Mean \pm SEM, $n=6,{ }_{p} a_{p}<0025,{ }_{p}<0.10, c_{p}<0.025, d_{p}<0.005$, $\left.\mathbf{e}_{\mathbf{p}<0.05}, \mathbf{f}_{\mathbf{p}<0.0005}, \mathrm{~g}_{\mathbf{p}}<0.001\right)$

\begin{tabular}{|c|c|c|c|}
\hline Parameters & Group I & Group II & Group III \\
\hline TLC $\left(10^{3} / \omega \mathrm{L}\right)$ & $6.8 \pm 0.27$ & $7.13 \pm 0.09$ & $7.62 \pm 0.36$ \\
\hline NEUROPHILS (\%) & $56.5 \pm 0.61$ & $56.5 \pm 0.53^{\mathrm{a}}$ & $46.7 \pm 0.66^{\mathrm{a}}$ \\
\hline LYMPHOCYTE $(\%)$ & $32.5 \pm 0.62$ & $27.4 \pm 0.31^{\mathrm{a}}$ & $39.18 \pm 0.83^{\mathrm{b}}$ \\
\hline MONOCYTES (\%) & $5.53 \pm 0.51$ & $6.73 \pm 0.38$ & $6.68 \pm 0.49$ \\
\hline EOSINOPHILS (\%) & $0.55 \pm 0.18$ & $5.78 \pm 0.4^{\mathrm{b}, \mathrm{h}}$ & $3.16 \pm 0.35^{\mathrm{b}}$ \\
\hline BASOPHILS $(\%)$ & $0.25 \pm 0.03$ & $1.32 \pm 0.26^{\mathrm{b}}$ & $1.6 \pm 0.42$ \\
\hline Hb $(\mathrm{g} / \mathrm{dL})$ & $11.53 \pm 0.41$ & $13.5 \pm 0.43^{\mathrm{g}}$ & $14.7 \pm 0.47^{\mathrm{i}}$ \\
\hline PVC $(\%)$ & $39.08 \pm 1.42$ & $43.7 \pm 0.4$ & $42.18 \pm 0.82$ \\
\hline MCV (FL) & $93.05 \pm 0.69$ & $97.3 \pm 0.45$ & $98.9 \pm 0.66^{\mathrm{e}}$ \\
\hline MCH (pq) & $30.7 \pm 0.48$ & $27.1 \pm 0.55^{\mathrm{c}, \mathrm{b}}$ & $31.5 \pm 0.76^{\mathrm{c},}$ \\
\hline MCHC (g/ dL) & $31.2 \pm 0.51$ & $33.5 \pm 0.64$ & $33.7 \pm 0.52^{\mathrm{f}}$ \\
\hline PC $\left(10^{3} / \mu \mathrm{L}\right)$ & $339.18 \pm 0.78$ & $373 \pm 6.08^{\mathrm{d}}$ & $350.5 \pm 1.87^{\mathrm{d}, \mathrm{g}}$ \\
\hline
\end{tabular}


Results are expressed as Mean \pm SEM values carrying different superscripts from the control for each parameter are significantly different from the experiments, where $\mathrm{n}=6$, and statistical significance was considered to be $\mathrm{a}_{\mathrm{p}<0.0025,} \mathrm{~b}_{\mathrm{p}<0.10,} \mathrm{c}_{\mathrm{p}<0.025,} \mathrm{~d}_{\mathrm{p}<0.005}$, $\mathrm{e}_{\mathrm{p}<0.05,} \mathrm{f}_{\mathrm{p}<0.0005,} \mathrm{~g}_{\mathrm{p}<0.001}$.

Assessment of hematological parameters can not only be used to determine the extent of deleterious effect of extracts on the blood of an animal, but it can also be used to explain blood relating functions of a plant extract or its products [13].

An increase in the number of WBC is a normal reaction of rats to foreign substances, which alter their normal physiological processes.

Platelets play a major role in the development as well as in the stability of atherosclerotic plaques and as a consequence, anti-platelet agents have been used clinically in patients at risk for myocardial ischemia, unstable angina and acute myocardial infarction [3].

The hemoglobin content, RBC and PCV has also significantly increased stimulate erythropoietin release in the kidney which is the humoral regulators of $\mathrm{RBC}$ production $[14,15]$. The aqueous extract of $P$. guajava had significant effect on $\mathrm{RBC}, \mathrm{Hb}, \mathrm{MCHC}, \mathrm{MCH}, \mathrm{PCV}, \mathrm{MCV}$, neutrophils, basophils, monocytes, lymphocytes and eosinophils.

The WBC was significantly elevated $(\mathrm{p}<0.05)$ in the group treated with 250 $\mathrm{mg} / \mathrm{kg}$ body where as the count significantly decreased $(\mathrm{p}<0.05)$ in $500 \mathrm{mg} / \mathrm{kg}$ body weight. The platelet also stimulate erythropoietin release in the kidney which is the humoral regulators of $\mathrm{RBC}$ production $[14,15]$.
Table2: Effects of Terminalia arjuna's bark extracts on immunoglobulin profile of male albino wistar rats (Mean \pm SEM, $\left.\mathrm{n}=6,{ }^{\mathrm{a}} \mathrm{p}<0.025,{ }^{b_{p}}<0.05,{ }^{c_{p}}<0.10\right)$

\begin{tabular}{|l|l|l|l|}
\hline Parameters & Group I & Group II & Group III \\
\hline IGG(gm/l) & $2.54 \pm 0.29$ & $2.96 \pm 0.15$ & $2.91 \pm 0.12$ \\
\hline IGM(gm/l) & $2.72 \pm 0.33$ & $0.63 \pm 0.27^{\mathrm{b}}$ & $0.5 \pm 0.1^{\mathrm{c}}$ \\
\hline IGA $(\mathbf{g m} / \mathbf{l})$ & $2.84 \pm 0.26$ & $2.45 \pm 0.25^{\mathrm{a}}$ & $2.64 \pm 0.29^{\mathrm{a}}$ \\
\hline
\end{tabular}

Pronounced variation was observed in hematological profile had significant effect on TLC, neutrophils, lymphocyte, monocytes, eosinophils, basophils, $\mathrm{Hb}$, PVC, MCV, MCH, MCHC and on PC. The present study reported the effect of T.arjuna extract on hematological profile was found to be $(7.13 \pm 0.09),(56.5 \pm 0.53)$, $(27.4 \pm 0.31), \quad(6.73 \pm 0.38), \quad$ significantly increased $(\mathrm{p}<0.05)$ in rats treated with both the doses.

The hemoglobin content, RBC and PCV has also significantly increased (5.78 \pm 0.4$), \quad(1.32 \pm 0.26), \quad(13.5 \pm 0.43)$, $(43.7 \pm 0.4),(97.3 \pm 0.45), \quad(27.1 \pm 0.55)$, (33.5 \pm 0.64$),(373 \pm 6.08)$ respectively.

While it showed significant increase in TLC, monocytes, eosinophil, monocytes, $\mathrm{Hb}, \mathrm{MCV}, \mathrm{MCHC}$, and $\mathrm{PC}$ in both the cases, when it is treated with low doses or with high doses but in neutrophil, PVC it significantly increased with low doses and reduced significantly with high doses and lymphocyte reduced with low doses and significantly increased with high doses. 


\section{Immunology}

The effect of the methanolic extracts of Terminalia arjuna was tested on albino male mice, it was indicated by the significant increase in IgG level in dose 50 $\mathrm{mg} / \mathrm{kg}$ that regulation of antibody production induced after 2 weeks of extract plant treatment, this increase in circulating IgG level is believed to contribute to B-cell function, it is believed that the presence of flavone glycosidic components in the plant extract of Terminalia arjuna [16] is responsible for this increase, this result was agreement with who found that methanolic extract of Anastatica hierochuntia significantly increased the serum levels of IgG, IgA and IgM of diabetic rats. These results are supported with those obtained by Nahla et al. [16] they stated that ethanolic extract of Cleome droserifolia significantly increased serum total protein, albumin, $\alpha$-globulin, $\beta$ - globulin and $\gamma$ globulin of rats, the increase may be an expression of the formation of more antibodies [16-18].

Pharmacognostical study of certain species of Anastatica. Ph.D. Thesis, Cairo University, Egypt) The IGG count showed no significant change in low dose, high dose as compared to control and non-significant changes as low dose, high dose compared. The IGM count showed significant changes $(\mathrm{p}<0.05)$ when low dose, control compared. Increased significantly $(\mathrm{p}<0.10)$ when high dose, compared to control and non- significantly changes observed in low dose, high dose comparison. The IGA count showed no significant changes when low dose, high dose was compared to control but increased significantly $(\mathrm{p}<0.25)$ when low dose compared to high dose.

\section{ACKNOWLEDGEMENT}

The authors acknowledge the facilities made available by the Department of Zoology, Ranchi University, Ranchi, for accomplishment of this work.

\section{REFERENCES}

1. Fang Xie., Chun FuWu., Wan-PingLai., $\mathrm{Xu}$ Juan Lang., Yuan Cheung. and XinSheng yahoo (2005), Evid Based Compl Altrn Med 2005, 2:3:353-361.

2. Karthikeyan, K., Bai Sarla, B.R., Gauthaman, K., Satish, K. S. and Devraj Niranjali S. (2003), Cardioprotective effect of alcoholic extract of Terminalia arjuna bark in an in vivo model of mycocardial ischaemic repurfusion injury, Life Sciences 73: 2727 - 2739 (2003)

3. Kullu, A., R., Tabassum, W., and Sinha, M. P. (2013) Effects of Psidium guajava aqueous extracts on hematological profile and serum lipid variables of albino rats, The Bioscan 8(2): 743-746.

4. Adebayo, J. O., Adesokan, A. A., Olatunji, L. A., Buoro, D. O. and Soladoye, A. O. (2005), Effect of ethanolic extract of Bougainvilea spectabilis leaves on hematological and serum lipid variables in Rats. Biokemistri 17(1): $45-50$.

5. Akhter, S., Hossain, I., Haque, A., Shahriar, M., Ahmed Bhuiyan, M. (2012), Phytochemical screening, antibacterial, antioxidant and cytotoxic activity of the bark extract of Terminalia arjuna. European Journal of Scientific Research 86(4): 543 - 552.

6. Kumar, M., Dandapat, S., Kumar, A. and Sinha M. P. (2013), Determination of nutritive value and mineral elements of Five-leaf Chaste tree (Vitex negundo L.) and Malabar Nut (Adhatoda vasica Nees). 
Academic Journal of Plant Sciences 6(3): $103-108$.

7. Kumar, M., Dandapat, S., Kumar, A. and Sinha, M. P. (2014), Pharmacological screening of leaf extract of Adhatoda vasica for therapeutic efficacy. Global Journal of Pharmacology 8(4): 494 - 500.

8. Kumar, M., Dandapat, S. and Sinha, M. P. (2015), Phytochemical screening and antibacterial activity of aqueous leaf extract of Punica granatum. Balneo Research Journal 6(3): 168 - 171.

9. Kumar, M., Dandapat, S., Sinha, M. P. (2014), Plant mediated synthesis of silver nano-particles using Adhatoda vasica aqueous leaf extract. The Ecoscan Special issue (5): $29-36$.

10. Kumar, M., Dandapat, S. and Sinha, M. P. (2014), Effects of Vitex negundo aqueous extracts on serum lipid variables and hematological profile of albino rats. The Ecoscan special issue (5): 135 - 140.

11. Kumar, M., Dandapat, S. and Sinha, M. P. (2015), Hepatoprotective activity of Adhatoda vasica and Vitex negundo leaf extracts against carbon tetrachloride induced hepatotoxicity in rats. Advances in Biological Research 9(4): 242 - 246.

12. Rifa, N. and Warnick, G. R. (2006), Lipids, lipoproteins, apolipoproteins and other cardiovascular risk factors. Text book of clinical chemistry and molecular diagnostics, $4^{\text {th }}$ edition, New Delhi: Elsevier pp. $942-960$.

13. Yakubu, M. T., Akanji, M. A. and Oladiji A. T. (2007), Alterations in serum lipid profile of male rats by oral administration of aqueous extract of Fadogia agrestis stem. Res J Med Plant 2: 66-73.

14. Degruchy, G. C. (1976), Clinical haematology in medical practice. Blackwell scientific publication. Oxford London.

15. Polenakovic, M. and Sikole, A. (1996), Is erythropoietin a survival factor for red blood cells? Journal of American Society of Nephrology 7(8): $1178-1182$.

16. Nahla, S. S. and Abdel-Nabi. I. M. (2006), Hypoglycemic effect of Cleome droserifolia ethanolic leaf extract in experimental diabetes and on non-enzymatic antioxidant, glycogen, thyroid hormone and insulin levels. Diabe-tologia Croatica. 35: 15-22.

17. Sherifa H. S., S. A. Halima, S. A. Amal and E.A. Abdel-Rahim. (2011), The antioxidative effects of some medicinal plants as hypoglycemic agents on chromosomal aberration and abnormal nucleic acids metabolism produced by diabetes stress in male adult albino rats. Journal of Diabetes Mellitus. Vol.1, 1: 614. 19.

18. Khalifa, T.A. (1980). Pharmacognostical study of certain species of Anastatica. Ph.D. Thesis, Cairo University, Egypt) 